\title{
Estudio filantrópico del Modelo Pedagógico de Praxeología de Uniminuto: aproximación analógica a nivel socio-educativo
}

\section{Philanthropic study of the Pedagogical Model of Uniminuto Praxeology: analogue approach at socio-educational level}

\section{ÓscarJavierZambranoValdivieso'-PabloLleralLaraCalderón²-AlejandraMaríaGutiérrezEspalza \\ (iD) http://orcid.org/0000-0003-0064-1062 - ${ }^{2}$ (iD http://orcid.org/0000-0002-8306-3861 iD http://orcid.org/0000-0002-6213-5846 \\ Corporación Universitaria Minutode Dios,Bucaramanga,Colombia}

Resumen

Objetivo: Realizar un estudio filantrópico del Modelo Pedagógico de Praxeología de Uniminuto como aproximación analógica a nivel socio-educativo.

Método: Se presenta una revisión de aportes al tema de autores como Bauman (2007) desde su obra Los retos de la educación en la modernidad líquida: Bedoya (2008) con Pedagogía ; Enseñar a pensar? Carbonell (2008) desde Una educación para mañana, Caride (2009) a partir del Elogio de la pedagogía social: acerca de los nuevos y viejos desafíos de la educación social, Hincapié (2012), entre otros.

Resultados: Al analizar el sector de la educación, es interesante cómo el aspecto pedagógico interactúa con lo social para pretender mejorar la calidad de vida del ser humano, la educación, para ser pertinente, debe preparar a los ciudadanos del futuro para un proceso de aprendizaje, continuo y autónomo, a lo largo de su vida; además, debe promover el trabajo multidisciplinario, la integración apropiada y crítica de las tecnologías, el dominio de otros idiomas, incentivar la disciplina, la dedicación, la fijación de metas y el sentidocrítico.

Conclusiones: El Modelo Pedagógico de Praxeología de Uniminuto permite formar un ser humano integral, capacitado en tratar los principios teóricos y llevarlos en transición a la praxis como acto educativo, dicho proceso permitirá que cada estudiante se traduzca en el epicentro de los cambios socio-educativos en términos micro locales, respondiendo a una educación ribosómica, con múltiples dimensiones dirigidas a transformar vidas, resimbolizando los imaginarios colectivos.

Palabras clave: Praxeología, Pedagogía, Educación, UNIMINUTO

Abstract

Objective: Carry out a philanthropic study of Uniminuto's Pedagogical Model Praxeological as an analogical approach at a socio-educational level.

Method: A review of contributions to authors such as Bauman (2007) is presented from his work The challenges of educationinliquidmodernity; Bedoya (2008) with Pedagogy Teachtothink?, Carbonell (2008) from An education for tomorrow; Caride (2009) from the Praise of social pedagogy: about the new and old challenges of social education, Hincapie (2012), among others.

Results: When analyzing the education sector, it is interesting how the pedagogical aspect interacts with the social to pretend to improve the quality of life of the human being, education, to be relevant, must preparethecitizens of thefutureforalearningprocess, continuousandautonomous,throughout his life; in addition, it should promote multidisciplinary work, appropriate and critical integration of technologies, mastery of other languages, encourage discipline, dedication, goal setting and critical thinking.

Conclusions: The Uniminuto Model Pedagogical Praxeology allows as an educational system to train an integral human being, trained in dealing with theoretical principles and take them in transition to praxis as an educational act, this process will allow each student to translate into the epicenter of changes socio-educational in micro local terms, responding to a ribosomic education, an education with multiple dimensions aimed at transforming lives, resimbolizing collective imaginaries.

Key words: Praxeology, Pedagogy, Education, UNIMINUTO.

Cómo citar este artículo(APA):

Zambrano, O., Lara, P.\& Gutiérrez, A. (2018). Estudio filantrópico del modelo pedagógico de praxeología de Uniminuto: aproximación analógica a nivel socio-educativo. Revista Educación y Humanismo, 20(34), 187-200.DOl: http://dx.doi. org/10.17081/eduhum.20.34.2864
Open Access

Editor:

Patricia Martínez Barrios Universidad Simón Bolíva

Correspondencia: Oscar Zambrano Valdivieso ozambranov1@uniminuto. edu.co

Recibido: 10-12-16 Aceptado: 30-04-17

Publicado: 01-01-18

DOI: http://dx.doi org/10.17081 eduhum.20.34.2864 


\section{PLANTEAMIENTO DEL TEMA}

El estudio filantrópico del modelo pedagógico de Praxeología de UNIMINUTO, permite desarrollar una aproximación analógica a nivel socio-educativo, entendiendo que el problema subyacente en la mayoría de las sociedades del mundo es la incapacidad de desarrollar modelos educativos propios que respondan a patrones culturales en contexto, los cuales a su vez puedan introspectivamente sistematizar la experiencia científica en el campo pedagógico que desarrollan en el transcurrir del tiempo histórico. La capacidad de llenar los vacíos historiográficos en términos de la episteme pedagógica constituye un método de abordar la praxeología como un modelo que responde a necesidades filantrópicas, evidencia un paradigma emergente que irrumpe coyunturalmente los modelos tradicionales, los cuales han migrado constantemente de contextos a otros contextos variando los métodos de adecuación social.

En este mismo orden de ideas es perentorio crear un arco conceptualizador que permita entender filosóficamente la praxeología, ante ello es fundamental que la existencia académica del maestro y del estudiante coexistan en el apostolado moral en el cual ambos en esa sinergia de realimentación intelectual, no debe ceñir su conductualidad a un mero acto cognitivo de almacenar información o de transición a lo cognoscitivo que vendría siendo el procesamiento de los datos para dar como resultado la construcción de un criterio en yuxtaposición. Todo lo contrario, la praxeología genera un estado de trascendencia epistemológica donde el conocimiento del docente debe transformar la calidad de vida, emancipándolo a través de la educación y el estudiante deberá transformar paulatinamente la realidad donde se desenvuelve, es decir, en la medida que se culturiza debe culturizar a la microsociedad con la adquisición de herramientas que permitirán proyectualmente resimbolizar la realidad tácita.

Con base a los planteamientos anteriores surge la necesidad de citar a Vargas (2013) quien nos refuerza el planteamiento al decir que la educación, como hecho cultural, se puede mirar de múltiples formas: como suceso histórico, como proceso de formación humana, como los modos de vivir y de pensar que deben ser como el acervo de bienes culturales a transmitir y a recrear. La principal característica de la educación: la apertura a corrientes de pensamiento.

El autor antes citado nos conecta con las corrientes de pensamiento que nacen en escuelas de pensamiento inherentes en términos universales al Positivismo, el Materialismo Histórico, el Humanismo, el Constructivismo, las 
cuales en algunos casos se originaron como teorías y se transformaron en corrientes de pensamiento, al punto que hoy exista una corriente principal del conocimiento donde tienen cabida aquellos modelos de pensamiento y acción que responden a necesidades filantrópicas o científico orgánicas propias de las sociedades actuales.

El modelo praxeológico en su dinámica se vuelve ecuménico ya que no niega la importancia de todas las corrientes, siempre y cuando todo el acervo transite de la teoría a las prácticas transformadoras para el bienestar humano, para ello necesita un alto nivel formativo a nivel presencial o virtual, donde la socialización es posible en algunos programas por la posibilidad de encontrase maestro y estudiante en tiempos y espacios comunes, en otras situaciones de contexto la socialización intelectual será generada por la tecnología a través de plataforma, el medio para la educación del ser humano es socio-tecnológico pero el fin es que a través de un acto creativo sapiencial el estudiante egresado recurra a sus herramientas filantrópicas y científicas para generar nuevos mecanismos evolutivos sociales, donde lo que se aprende no queda en lo mero sistémico sino se convierte en trans-sistémico.

El estudio filantrópico del modelo pedagógico de praxeología de UNIMINUTO: se basa en el objetivo que la educación llegue a los colombianos de cualquier estrato social y de cualquier espacio constitutivo de la geografía del territorio colombiano, estas intenciones socio-educativas se enmarcan en dos sistemas curriculares, uno en el marco tradicional de la presencialidad y el otro bajo los cánones emergentes de la educación virtual.

Los dos sistemas educativos antes mencionados referidos a la presencialidad y a la virtualidad conllevan a que se genere un proceso socio-educativo interesante ya que la presencialidad tiene el reto de introducir los recursos de la tecnología de la información y comunicación como componente didáctico constante en la enseñanza de los estudiantes, para que ellos se desarrollen intelectualmente a la par con las exigencias del mundo globalizado o tecnificado universalmente.

En el caso de la virtualidad se presentan como exigencias stricto sensu, el manejo de la plataforma modle 3.1, una atmósfera socio-tecnológica en el cual el ser humano puede desarrollarse con un pensamiento crítico a partir de componentes de tecnología dirigida a crear puentes didácticos para el acercamiento entre el estudiante y el maestro. Este planteamiento arrastra en consecuencia una nueva socialización comunicativa de la educación ya 
no desde el formato griego del manuscrito heredado hasta la actualidad, sino a través de interacciones de producción intelectual digital, que va desde un manejo de la episteme a maximis ad minima. El sistema praxeológico es un cónclave basado en principios de autotransformación y transformación de vidas colombianas, todo ser humano para emanciparse no puede desligarse de la educación, es por ello que la enseñanza sistemáticamente puede coadyuvar que los habitantes de cualquier estrato puedan desarrollarse intelectualmente, comprendiendo que el acto formativo construye psíquicamente las herramientas para que el ser humano desarrolle potencialidades como constructo de vida.

El sistema de enseñanza virtualizado implica como reto didáctico romper la superestructura tecnológica, esto se logra en la medida que se logre humanizar las plataformas donde interactúan el preceptor con el aprendiz, el maestro con el alumno, el docente con el estudiante o el orientador con el discente, cual sea la relación filantrópica, en dicha estructura es inextricable la necesidad de introducir elementos que funcionalmente permitan que los procesos de socialización del conocimiento se perciba en tiempo real, es por ello que es fundamental construir un puente teórico riguroso que genere la transición teórico-empírica en un proceso horizontal de integración entre la teoría y la práctica, desde cualquier espacio de aprendizaje ya sea físico o virtual. Cabe agregar sustantivamente que la praxeología desde la dimensión pedagógica se manifiesta como un marco conceptual integrador de la educación, presencial o virtual, que se adhiere a valores humanistas fundamentales, pero que invita tanto a los formadores como a los aprendices, a un proceso reflexivo y crítico sobre su propia práctica, en el marco de una real flexibilidad curricular.

Desarrollar un proceso de transición efectiva entre la teoría y la praxis en cualquier espacio socio-educativo en el que se dinamice el discente, implica que se inocule de principios filantrópicos circunscriptos en la psicología positiva activa de la motivación, en el que el discente aplique lo aprendido en términos transformadores de la sociedad inmediata, generando nuevos escenarios resimbolizados didácticamente para que los niños y jóvenes de esa microsociedad conciban su espacio como un lugar que se puede sembrar mejoras con las cuales en el transcurrir del tiempo, dichos grupos humanos se sientan satisfechos con el mejoramiento paulatino del paisaje cultural en el que se mueven, entendiendo que sus vidas alcanzan niveles altos de calidad en la medida que se emancipan con la educación. 
En los marcos de las observaciones anteriores se hace ineludible citar a Vargas (2010), quien tiene una perspectiva sobre la pedagogía praxeológica al referirse sobre ella de la siguiente manera: la praxeología no es un método pedagógico; ella, es más bien una visión, un ideal a conseguir y un marco integrador de la formación a ofrecer a los estudiantes y demás actores del proceso educativo. Según se ha citado el autor nos contextualiza en una dimensión de la praxeología no metódica sino filosófica basada en el para qué, el por qué y hacia dónde, esta perspectiva analítica permite observar claramente que la praxeología tiene una concepción integral del ser humano moviéndose en dos categoría de análisis: la primera el imaginario del sujeto que implica el método de abordaje de la realidad, y el segundo la imaginación línea en la cual el sujeto idealiza cómo podría cambiar esa realidad en la cual ha estado sumergido toda la vida o los procesos de aculturación lo sumergió en un determinado marco temporal de la sociedad en la que se desenvuelve.

A lo largo de los planteamientos hechos hemos expuesto las distintas aristas de la praxeología en sus distintos pisos de compresión analítica, con base a ello es fundamental fortalecer nuestro discurso socio-educativo y para ello procedemos a citar a Bernabéu (1997), quien nos afirma que la educación es una acción social orientada hacia unas finalidades en la que se realizó una influencia, deliberada e intencional, sobre los sujetos para ayudarles a realizar unos proyectos individuales y sociales. Partiendo de este postulado educacional es pertinente decir con propiedad que el fundador de UNIMINUTO el padre Rafael García Herreros visualizó la praxis educativa como una acción crítica cotidiana, donde la dialéctica se convierte en instrumento para suprimir la imposibilidad de surgimiento de jóvenes con limitaciones económicas, en posibilidades para reproyectar sus vidas con proyectos para asegurar un mejor futuro desde la individualidad pensante, como desde la realidad construida por la masa social. La Universidad Minuto de Dios como sistema social confluye en la idea de formar a un ser integral, que va más allá de las ciencias formativas, transita a lo que hemos denominado las ciencias de la cotidianidad, que es donde el hombre formado en UNIMINUTO de forma pragmática es capaz de ordenar su conocimiento originando creativamente ideas que puedan propiciar soluciones reales a su espacio de necesidad que traspase el estado de fe estimulado por el padre Rafael García Herreros como legado comunicativo y transformador, desde 1950 hasta el año 2000.

La filosofía filantrópica del estudiante egresado debe basarse en la utilización de sus grandes ideas con transición a una praxis efectiva como podría ser alinear lo cognitivo identificando problemáticas de primera necesidad 
humana, como lo es tener que resguardarse y a partir de allí planear para lograr construir las casas necesarias para su barrio y abolir la miseria social. Un ser humano que representa la filosofía antes mencionada es el padre García Herreros, quien llegó a lograr las más de dos mil viviendas que hoy constituyen el barrio Minuto de Dios en Bogotá, o los cientos de viviendas en otras latitudes geográficas del país, en la medida que el ser humano se cultiva con educación, cultiva la metamorfosis social.

En este sentido y en términos conexos con lo antes expuesto es imperioso citar a García Herreros (s/a): "No solo es pegar ladrillos; es más fácil pegar ladrillos que pulir las piedras vivas que son los hombres en la construcción de una sociedad". En este orden de ideas se avizora con argumentos reales una nueva sociedad, posible con sistemas educativos integrales, dicha pedagogía social se viene gestando desde el siglo XX con unas excelentes perspectivas que hoy son manejadas y co-proyectables al futuro, no solo en palabras, sino también en hechos (Caride, 2009).

\section{REVISIÓN Y SITUACIÓN ACTUAL DEL TEMA}

Es interesante como el aspecto pedagógico interactúa con lo social para pretender mejorar la calidad de vida del ser humano. Caride, Gradaílle \& Caballo (2015) lo reafirman al explicar que cuando en las primeras décadas del tercer milenio lo pedagógico-social evocó la necesidad de abrir la educación a la vida, en toda su diversidad: entendiendo que esto significaba una aventura apasionante, de largos recorridos semánticos y pragmáticos, donde lo cotidiano se teorizó y fue puesto a la orden del día para que la sociedad se entendiera a sí misma para comenzar a plantearse un progreso antropocéntrico redignificando al hombre como motor propulsor de los avances integrales.

En la sociología se concibe al individuo y a la sociedad como una relación indisoluble, comprendiendo que el individuo es la representación del todo social y la sociedad representa en su conformación la individualidad del ser, es por ello que Carbonell (2008, p.19) concibe la educación de la vida y por la vida, como un "viaje simultáneo a la realidad y al interior de cada persona", inacabado e inacabable. La educación de todos y para todos, en su versión más cívica y cotidiana, emocional y cognitivamente desarrollada.

La educación adentrada al espíritu social permite que los conocimientos contemplados en las ciencias puras, en las Ciencias Sociales, se reconviertan desde un espíritu epistémico hacia una aplicabilidad emocional, entendiendo que en el siglo XXI los conocimientos son procesados y direccionados por las 
inteligencias múltiples, entre las cuales podemos nombrar la intrapersonal, la interpersonal, la emocional, la espiritual, sin obviar las inteligencias conducentes a formaciones académicas como la naturalista, la lingüística, la lógica matemática, en este mismo sentido el tratamiento que le da las inteligencias antes enunciadas tienen un procesamiento praxeológico ya que permite encontrar canales cognoscitivos para la resolución de problemáticas individuales o colectivas de la vida social ya conformada como sistema educativo para la vida.

El conocimiento según la nueva tendencia es transmitida por herencia genética, en la mayoría de los casos el conocimiento se produce por transmisión oral o escrita en actos magistrales o cotidianos de enseñanza con el uso de nuevas o tradicionales técnicas didácticas que generan en el ser humano la posibilidad de conocerse como ente bio-psico-social, este planteamiento es respaldado por Esteve (2010), quien plantea que el proceso introspectivo de conocerse como ser humano y las implicaciones socio-históricas que las mismas tiene, comienza en el mismo momento del nacimiento, el cual debe conducir a que el ser humano se comprenda y entienda a sí mismo y al mundo que lo rodea, procurando que sea plenamente partícipe del complicado don de la convivencia. En el proceso de estudio mediante el modelo praxeológico con una concepción filantrópica, el ser humano define su sentido de coexistencia social, con base a esta causalidad existencial el individuo en rol de estudiante comprenderá cuál debe ser su propósito en su desempeño profesional, se trabaja para que el ser humano se conciencie en una educación para la vida o en lo que plantea Innerarity (2013), quien dice que el hombre tiene que desarrollar una educación puesta al servicio de las personas y del desarrollo humano.

Así mismo el autor anteriormente referenciado afirma la sensibilidad que ha de tener la educación hacia las necesidades de todos, pero muy especialmente de quienes son rechazados por los modelos de producción y consumo inducidos por los mercados, como se había ya afirmado anteriormente; ahora, en el mundo de todos y de nadie que habitamos (Innerarity, 2013). La educación de la vida permite que las microsociedades evolucionen sin la inherencia de super estructuras de mercados que marcan las pautas de comportamiento universal, donde condiciona la relación bilateral entre lo que debe ser la educación a partir de tendencias unívocamente económicas y lo que podría ser a partir de la definición de necesidades propias y en contextos direccionadas con autonomía. 
Las sociedades de Europa, Latinoamérica o de cualquier continente han cambiado su estructura debido a que han llegado a un punto de disfuncionalidad, a partir de esta premisa las sociedades recrean necesidades y objetivos para mantenerse estables, produciéndose las transiciones desde una sociedad antropocéntrica a una teocéntrica, a una heliocéntrica o a una holística como sucede en los actuales momentos.

La sociedad holística se caracteriza por tener unas implicaciones formativas de impacto, en otras palabras, la formación que el ser humano recibe en la actualidad debe traducirse en las herramientas indispensables para afrontar lo impredecible del futuro, en esta postura analítica, también se posiciona Bauman (2007, p.47), igualmente por Caride (2009), señala que nunca antes se encontraba la sociedad en una situación semejante, en la que no solo se debe aprender el arte de vivir en un mundo sobresaturado de información, "también debemos aprender el aún más difícil arte de preparar a las próximas generaciones para vivir en semejante mundo". En palabras de Padilla (2015), la pedagogía actual es un llamado a ver la realidad como un gran sistema, que debe ser analizado desde una perspectiva educativa en beneficio del desarrollo social. De esta manera, es importante determinar la pedagogía como el saber dicotómico donde coexiste la teoría y la praxis. La segunda mitad del siglo XX y las primeras décadas del nuevo milenio serán registradas por la historia como un tiempo de considerables transformaciones. Dichos cambios han acompañado el desarrollo de la educación, incitándolo en algunos casos, en otros retardándolos, pero siempre visibilizando giros significativos en los sistemas educativos y los enfoques pedagógicos (PEI UNIMINUTO, 2014).

Hoy se habla de apertura, globalización, internacionalización, mundialización, sociedad de la información y del conocimiento, sociedad post-capitalista, sociedad post-industrial, nueva edad media, tercera ola, modernidad líquida, expresiones acotadas por autores como Jacques Delors, Peter Drucker, Alain Minc, AlvinToffler, Toni Negri y Zygmunt Bauman, entre otros autores, dichas acotaciones, evidencian la intercomunicación en tiempo real de todas las sociedades del mundo, transfiriéndose sus nuevas producciones científicas, intelectuales, nutricionales, que intentan crear en todas las latitudes del globo terráqueo il uomo di vitruvio, hombre universal que se le provea las mismas herramientas para la vida, intentándose generar un nuevo escenario donde el individuo no conflictúe con la vida sino que se integre a ella. UNIMINUTO reconoce esta realidad y por ello comparte su preocupación por mejorar permanentemente el proceso de enseñanza-aprendizaje y sobre 
todo por lograr resultados orientados a ofrecer profesionales con la experticia necesaria para dar soluciones en la medida de los recursos escasos a las necesidades básicas y holísticas de la sociedad, a partir de un saber que se convierte en praxis.

Todo ello anuncia un cambio en la construcción de las dinámicas sociales y de los sujetos, en las formas de relación entre los individuos y en los modos de aprender y de administrar el conocimiento. En este panorama, la Corporación Universitaria Minuto de Dios UNIMINUTO, en su proyecto educativo institucional refuerza el carácter e importancia de la sociedad y acorde con Ritzer (1997, pp.8-9), comparte que las características principales de la sociedad actual son: a) Crítica radical al fracaso de la Modernidad (a la Razón como absoluto). b) Rechazo de toda explicación unitaria y coherente (cosmovisiones, metarrelatos, utopías). c) Énfasis en lo premoderno: emoción, sentimiento, meditación, magia, rituales, mitos. d. Desafío a los límites modernos, como modo de organizar el mundo: definiciones, barreras entre disciplinas, diferencia entre realidad y ficción. e. Atención especial a la periferia de la sociedad, no a su centro; características que se deben revisar constantemente para mejorar el proceso de la praxeología social.

Bajo este contexto mundial, desde hace varias décadas, la sociedad colombiana se viene transformando. Las cosmovisiones de los colombianos de hoy son muy diferentes de las vigentes hace dos o más décadas. Las formas de pensar y actuar han cambiado hasta niveles insospechados debido al apresurado proceso de urbanización, al incremento absoluto de la población, al aumento de la esperanza de vida y de los niveles de alfabetización y educación, a la disminución de las distancias, a la transculturación internacional y a la creciente incidencia de los medios de comunicación de masas (PEI UNIMINUTO, 2014).

En palabras de Martínez (2008:121), "En nuestro contexto sociocultural y político occidental y en vías de desarrollo en el que vivimos, la educación debe procurar que todos y cada uno de los ciudadanos y ciudadanas seamos competentes para comprender nuestro mundo, convivir juntos y actuar con criterio. Este cambio acelerado ha modificado estructuralmente las instituciones sociales alterando la forma y el contenido de las relaciones personales, y cuestionando estructuras que durante años sirvieron de fundamento a la sociedad como la familia, la iglesia, la escuela y los partidos políticos. En otras palabras, se han trastornado las instancias que posibilitan el juego de fuerzas que luchan por lograr la dirección ética, intelectual y moral de la 
sociedad; es lo que comúnmente se llama «crisis de valores».

Hay que destacar que este proceso, por las características sociales, étnicas, geográficas y de diverso orden que se han dado en el país a lo largo de su historia, ha determinado la aparición de desarrollos culturales regionales diferenciados, aunque ligados a un mismo acervo histórico. Respetando las diferencias culturales, la identidad cultural ha de ser planteada como un común proyecto socio-histórico, una educación orientada a converger esfuerzos para crear condiciones que permitan a las generaciones más jóvenes adquirir las competencias necesarias para poder vivir de manera sostenible y sustentable tanto a nivel personal, laboral, como también en el ámbito comunitario. En este contexto corresponde abordar la formación para la ciudadanía y en él adquiere sentido como uno de los objetivos claves de la tarea educadora y de las políticas educativas (Martínez, 2008).

La sociedad colombiana presenta un desarrollo educativo que está beneficiando a la comunidad más vulnerable y UNIMIMUNTO hace parte de esta realidad (PEI UNIMINUTO, 2014). Tras un atraso sorprendente hasta mediados del siglo XX, la educación tuvo un significativo impulso durante los años 60 hasta mitad de los 70 del siglo pasado. Ahí el ritmo de crecimiento educativo se redujo, entre otras causas, por problemas institucionales (centralismo, falta de coordinación e información y ausencia de participación). Dichos problemas se empiezan a corregir con el nuevo marco constitucional que propende por la descentralización educativa, por una mayor organización, coordinación y gestión a nivel regional, y una participación activa de la comunidad en la dirección de las instituciones de educación (Constitución Política de Colombia, 1991, Art. 68), mediante juntas educativas y manejo presupuestalpropio.

Una política educativa colombiana acertada debe partir de la premisa de la universalización del ingreso a un sistema educativo que ofrezca a sus graduados el conocimiento, las competencias y la autonomía requeridas para enfrentar los retos del entorno económico global, los contextos y realidades del desarrollo nacional como también los cada vez más sofisticados espacios de interacción social. La educación, para ser pertinente, debe preparar a los ciudadanos del futuro para un proceso de aprendizaje, continuo y autónomo, a lo largo de su vida; además, debe promover el trabajo multidisciplinario, la integración apropiada y crítica de las tecnologías, el dominio de otros idiomas, incentivar la disciplina, la dedicación, la fijación de metas y el sentido crítico. Además, el profesional que se requiere hoy debe estar en capacidad de desempeñarse en áreas específicas, de innovar y ser suficientemente flexible, 
para responder a un entorno rápidamente cambiante. Pero, además, requiere que adquiera sensibilidad desde la filantropía y compromiso social partiendo de los principios resilientes (PEI UNIMINUTO, 2014).

Con base en la perspectiva analítica antes descrita tenemos un autor que refuerza los fundamentos pasados, nos referimos a Martínez (2008), quien expresa que un modelo formativo y de aprendizaje propio de la sociedad de la información debe estar orientado al logro de competencias en combinación con la adquisición de recursos cognitivos, para que el estudiante se dinamice con criterios definidos en contextos reales para generar situaciones de aprendizaje para el servicio como apostolado pedagógico. Zambrano (2006) afirma que la construcción del pensamiento pedagógico según Philippe Meirieu está centrado entre el decir y el hacer, donde se recogen las categorías de estudio y de análisis; deduce que en el bloque del decir se identifica la práctica reflexiva, que se entiende como aquel momento en el cual un sujeto está en capacidad de hacer la síntesis de lo que precisamente elabora en la dimensión del hacer.

La práctica como metodología didáctica de enseñanza tiene como conceptos fundamentales la experiencia, el sentido y la percepción. La primera informa lo que un sujeto alcanza en la práctica de su ser, el tiempo que presupone y la distancia que impone tanto en su espíritu como en las actividades que realiza; seguidamente, el sentido, concepto igualmente hermenéutico, permite comprender las orientaciones que toda actividad realizada por un sujeto mantiene en un horizonte de verdad. El sentido aparece como la dirección de lo que se hace y repercute en la dimensión espiritual de un sujeto. En términos conjeturales, la percepción informa el modo de ver de un sujeto, en cuya esencia se interrelacionan, a la vez, el sentido y la experiencia. Es así como Botero, Morán y Solano (2010), afirman que se debe formar a un hombre consciente de sí mismo, que actúe conforme a unos valores universales y de contexto en niveles éticos, morales y políticos, capaz de señalarse caminos y metas que superen su condición individual de vulnerabilidad y a partir de allí, formular y llevar a cabo iniciativas que contribuyan a la construcción de condiciones de vida más humanas, más dignas para las masas sociales.

El prototipo de hombre que UNIMINUTO ha perfilado como sujeto social, obedece a un constructo de experiencia sujecionadas a la sensibilidad telúrica inoculada en el espíritu formador del padre García Herreros y cofundadores del principio universal sobre desarrollar sistémicamente la pedagogía del Minuto de Dios y la didáctica de servir, en consecuencia Bedoya (2008), 
deduce que el concepto de formación, es la línea vertebral axiomática y que alrededor de él, se pueden seguir definiendo nuevos conceptos para formar desde la sensibilidad humana, procurando coartar la masificación de sociedades frívolas, que observan al sujeto como un mero objeto para la producción o para el desarrollo de sociedades hedonistas, este cuadro analítico forma parte del soporte conceptual que direcciona la investigación en Uniminuto para responder a la realidad heterogénea de Colombia.

Hincapié (2012, p.3) realiza un análisis muy significativo en su obra "La praxeología como alternativa socialmente responsable para la superación de la vulnerabilidad", en esta indica que la superación debe iniciarse con un trabajo desde la praxeología que reivindique a la persona consigo misma, de manera que trascienda aquellos factores que robustecen mental y actitudinalmente esa postura de constante socorro, para que luego se conciba como un actor que puede llevar a cabo un significativo cambio personal que repercuta en lo social (Schuster, 2008).

Además, Hincapié (2012) aduce que esto implica un quehacer praxeológico que en primera instancia encamine al sujeto hacia el reconocimiento de sí mismo, identifique las características de su temperamento, su carácter, sus fortalezas y debilidades, su alfabeto e inteligencia intra e interpersonal (Davies, 1994), en segunda, "aprenda a respetar las normatividades y las integre a un orden axiológico que le permita la adaptabilidad a la vida social” (Quintero, 2011, p.2) y en tercera, potencialice sus capacidades como líder para la consolidación de mejores relaciones interpersonales, de manera que con quienes hacen parte de su escenario sociogeográfico, conjuntamente puedan dar respuestas y soluciones a las problemáticas de su entorno (Duque, 2000; Hurtado, 2001; López, 2002).

\section{CONCLUSIONES}

El modelo pedagógico de praxeología de UNIMINUTO permite comosistema educativo formar un ser humano integral, capacitado en tratar los principios teóricos y llevarlos en transición a la praxis como acto educativo, dicho proceso permitirá que cada estudiante se traduzca en el epicentro de los cambios socio-educativos en términos microlocales, respondiendo a una educación ribosómica, una educación con múltiples dimensiones dirigidas a transformar vidas, resimbolizando los imaginarios colectivos.

El proceso de reestructuración de los arquetipos mentales del niño o joven colombiano concebido como ente bio-psico-social, se podrá desarrollar a 
partir de una cosmovisión filantrópica donde van a adquirir las herramientas para intervenir coyunturalmente su contexto y romper la estructura preexistente que lo condiciona a tener una tipología de vida, sin tener la posibilidad de transcender a otras dinámicas esenciales en el constructo personal.

La filantropía en el espectro del modelo praxeológico persigue desde la acción materializar las metas que desde niño se han esquematizado como utopías, este planteamiento se puede ejemplificar cuando el niño que sueña con ser un atleta de alta competencia en medio de la distribución de las drogas lo pueda hacer, que el niño que todas las noches escucha las balaceras en su comuna no le impida convertirse en científico de la NASA o llegar a ser un escritor mejor que Gabriel García Márquez, con otros elementos menos prosopopéyicos, lo que el modelo praxeológico busca es que el ser humano pueda vivir, sonreír y tener una vida emocional sana, realidad que puede ser cotidiana para muchos grupos humanos pero el ensueño para pueblos como los del norte de Bucaramanga y otras latitudes geográficas enajenadas por el oscurantismo regentando por los antisociales o terroristas espirituales, castradores de sueños recién encubados socio-educativamente.

El estudio filantrópico de la praxeología nos imbuye en un viaje introspectivo sobre el acto puro de educar, de cultivar el espíritu, la mente y las emociones de aquellos denominados vulnerables, de aquellos que son los más pobres, de los pobres que no tienen conciencia de lo pobres que son, los cuales son grupo emergente resultado de las acciones erróneas de unas minorías, al no romperse esa cadena sucesiva, los problemas humanos son incuantificables, cierro con la premisa que la educación no solo libera, sino te hace feliz, entendiendo la felicidad como un proceso de constante construcción y todo ser humano está obligado a ser de feliz capaz de autotransformarse y transformar la realidad del otro, que también sufre como tú y quiere ser libre de los estereotipos sociales que te matan estando vivos.

\section{REFERENCIAS}

Bauman, Z. (2007). Los retos de la educación en la modernidad líquida. Barcelona: Gedisa.

Bedoya, J. (2008). Pedagogía ¿Enseñar a pensar? Bogotá: ECOE. Bernabéu, J.L. (1997l). Educación y dimensiones de la educación. En Colombia. Iconos e instituciones contemporáneos de la educación. Barcelona: Ariel.

Botero, P., Morán, A. Y. \& Solano, R. (2010). Fundamentos conceptuales del CED. Educación para el desarrollo, formación ciudadana y responsabilidad social universitaria. Bogotá: Corporación Universitaria Minuto de Dios.

Carbonell, J. (2008). Una educación para mañana. Barcelona: Octaedro. 
Caride, J. A. (2009). Elogio de la pedagogía social: acerca de los nuevos y viejos desafíos de la educación social. Revista de Educacáo Pública, 18(38), 449-468.

Caride, J. A., Gradaílle, R. \& Caballo, M. B. (2015). De la pedagogía social como educación, a la educación social como Pedagogía. Perfiles educativos, 37(148), 4-11.

Constitución Política de Colombia (1991). Bogotá, Colombia: Leyer.

Davies, P. C. (1994). Dios y la nueva física. España: Salvat.

DIOS-UNIMINUTO, D. E. Corporación Universitaria Minuto (2014). PEI UNIMINUTO. Proyecto Educativo Institucional.

Duque, J. (2000). La misión de educar. Bogotá: Ediciones Eduque.

Echeverry, N. \& Junca. (2010). El objeto animado, una herramienta de la praxeología pedagógica. Tesis de grado. Universidad Minuto de Dios, Bogotá.

Esteve, J. M. (2010). Educar: un compromiso con la memoria. Barcelona: Octaedro.

Hincapié, J. M. M. (2012). La praxeología como alternativa socialmente responsable para la superación de la vulnerabilidad. Revista Poiésis, 12(24).

Hurtado, O. (2001). Cultura y desarrollo. Sociología del desarrollo, políticas sociales y democracia: estudios en homenaje a Aldo E. Solari. México: Siglo XXI.

Innerarity, D. (2013). Un mundo de todos y de nadie: piratas, riesgos y redes en el nuevo desorden global. Barcelona: Paidós.

López, A. (2002). El desarrollo de la vida personal. Revista Pensamiento y Cultura, 5(24), 69-81.

Martínez, M. (Ed.). (2008). Aprendizaje, servicio y responsabilidad social de las universidades. Barcelona: Octaedro.

Morillo Bentue, J. (2011). Praxeología: Definición. Recuperado de https:// www.juandemariana.org/ijm-actualidad/analisis-diario/praxeologia-definicion

Padilla, A. S. (2015). Retos de la pedagogía en el siglo XXI. Revista de Investigaciones UNAD, 13(2), 143-148.

Quintana, J. (1997). Antecedentes históricos de la educación social. En A. Petrus, Pedagogía Social (pp. 67-91). Barcelona: Ariel.

Quintero, H. H. (2011). La educación social y su opción para la intervención social en Colombia. Memorias, 9(15), 129-140.

Ritzer, G. (1997). Postmodern Social Theory. Nueva York: McGraw-Hill.

Schuster, H. A. (2008). El abrigo de un sueño "utopía realizada". Bogotá: CEIHS (Uniminuto).

Vargas, C. G. J. (2010). Hacia una pedagogía praxeológica. Departamento de Pedagogía. Bogotá: UNIMINUTO.

Vargas, C. G. J. (2013). Educación: un acercamiento praxeológico. Polisemia, (2), 13-38.

Zambrano Leal, A. (2006). El concepto pedagogía en Philippe Meirieu. Un modelo, un concepto y unas categorías para su comprensión. Revista Educación y Pedagogía, XVIII(44), enero-abril, 33-50. 